\title{
Intraperitoneal chemotherapy as adjuvant treatment to prevent peritoneal carcinomatosis of colorectal cancer origin: a systematic review
}

\author{
D A M Sloothaak ${ }^{1}$, B Mirck ${ }^{1}$, C J A Punt ${ }^{2}$, W A Bemelman ${ }^{1}$, J D W van der Bilt³, \\ A D'Hoore ${ }^{3}$ and P J Tanis ${ }^{*}, 1$ \\ ${ }^{1}$ Department of Surgery, Academic Medical Centre, Meibergdreef 9, 1105 AZ Amsterdam, The Netherlands; ${ }^{2}$ Department \\ of Medical Oncology, Academic Medical Centre, Meibergdreef 9, 1105 AZ Amsterdam, The Netherlands and ${ }^{3}$ Department of \\ Abdominal Surgery, Gasthuisberg Hospital, Herestraat 49, 3000 Leuven, Belgium
}

Background: Peritoneal carcinomatosis (PC) of colorectal cancer (CRC) origin is associated with poor outcome. This systematic review evaluates the available evidence about adjuvant (hyperthermic) intraperitoneal chemotherapy $((\mathrm{H})$ IPEC) to prevent the development of PC.

Methods: A systematic search of literature was conducted in August 2013 in PubMed, Embase, and the Cochrane database for studies on (H)IPEC to prevent PC in patients who underwent curative surgery for primary CRC.

Results: Seven comparative studies and five cohort studies were selected. Treatment schedules varied between repeated fluoropyrimidine-based IPEC administration in the ambulatory setting to intra-operative (H)IPEC procedures using mitomycin-C or oxaliplatin. The reported rates of major complications related to adjuvant (H)IPEC was low. Four out of five evaluable comparative studies reported a significant difference in the incidence of PC in favour of (H)IPEC. All three comparative studies reporting on survival after intra-operative (H)IPEC showed a significant survival benefit in favour of the experimental arm. Substantial heterogeneity in patient selection, treatment protocols, and treatment effect evaluation among studies was observed.

Conclusions: The currently available evidence about adjuvant (H)IPEC in high-risk CRC is limited and subject to bias, but points towards improved oncological outcome and supports further randomised studies.

The abdominal cavity is the second most common site of recurrence in patients with colorectal cancer (CRC) (Brodsky and Cohen, 1991). Peritoneal carcinomatosis (PC) is detected synchronously during primary resection in about $5 \%$ and develops metachronously in $4-19 \%$ of patients (Koppe et al, 2006; Lemmens et al, 2011; Segelman et al, 2012). The reported incidence of PC found at autopsy of patients who died from CRC ranges between 40 and $80 \%$ (Koppe et al, 2006). Because PC is difficult to diagnose, the true incidence of metachronous PC is not exactly known.
At the time PC is clinically manifest, only palliative treatment options remain in the majority of patients. However, modern systemic therapy is not as effective for PC as for liver metastases or other distant metastases (Franko et al, 2012; Klaver et al, 2012). A recent study reported a median survival for PC of CRC origin of 5 months if no chemotherapy was given, and 11-12 months for 5-fluorouracil/ leucovorin $(5 \mathrm{FU} / \mathrm{LV})$ and oxaliplatin/irinotecan-based systemic chemotherapy (Pelz et al, 2010). In selected patients with PC of CRC origin, cytoreductive surgery (CRS) with hyperthermic intraperitoneal chemotherapy (HIPEC) offers the only curative

\footnotetext{
*Correspondence: Dr PJ Tanis; E-mail: p.j.tanis@amc.uva.nl
}

Received 14 March 2014; revised 20 May 2014; accepted 9 June 2014; published online 15 July 2014

(c) 2014 Cancer Research UK. All rights reserved 0007-0920/14 
treatment option. The efficacy and the morbidity of this multimodality treatment highly depends on the extent of peritoneal dissemination, particularly patients with low-volume peritoneal disease and no evidence of systemic spread benefit from CRS/HIPEC (Verwaal et al, 2003; Glehen et al, 2004; Elias et al, 2009; Honore et al, 2013).

It seems more effective to treat patients with a high risk of developing PC, such as pT4 or perforated stages II or III CRC. Prophylactic (adjuvant) (H)IPEC might prevent the outgrowth of residual intraperitoneal tumour cells into macroscopic PC in such patients (Hompes et al, 2012; Honore et al, 2013). Several investigators have tried to treat PC at a clinically occult stage starting in the 1980s (Speyer et al, 1981). Adjuvant intraperitoneal 5 FU showed a variable efficacy and did not gain any interest in the oncological community for several years. More recently, success of $\mathrm{CRS} / \mathrm{HIPEC}$ in treating macroscopic PC renewed the interest in the potential role of adjuvant (H)IPEC. The aim of this systematic review is to evaluate the evidence that is currently available for adjuvant (H)IPEC to prevent PC in high-risk CRC patients.

\section{MATERIALS AND METHODS}

Search strategy. In August 2013, a systematic search of published literature was conducted in PubMed, Embase, and Cochrane databases. Combinations of the following search terms were used to identify relevant studies: 'intraperitoneal chemotherapy', 'intraperitoneal 5fu', intraperitoneal chemoperfusion', 'epic', 'hipec', 'advanced colon cancer', 'advanced CRC', 'peritoneal neoplasms', 'peritoneal metastases', 'peritoneal metastasis', 'cytoreductive', 'resection', 'surgery' (Supplementary Information I). No language or publication restrictions were applied.

Selection criteria. Titles and abstracts were screened for eligibility by two independent researchers (BM and DAMS). All studies on intraperitoneal chemotherapy as adjuvant treatment for CRC were considered eligible; other malignancies including appendiceal tumours were excluded. Duplicates, conference abstracts, studies about treatment of macroscopic PC instead of prevention of PC and animal studies were excluded as well. In case of disagreement about abstracts, the full text was examined and a decision for eligibility was made in consensus between the two researchers. In case of overlapping cohorts, full texts were obtained to select the most informative article for inclusion. Studies were considered eligible for inclusion if primary data were provided, regardless of the described end point (e.g., feasibility, toxicity, or survival).

Data analysis and quality of included studies. Methodological quality of the studies was assessed by two independent researchers (BM and DAMS) based on the quality assessment tools provided by the Dutch Cochrane Centre (Dutch Cochrane Center, 2014a, b). Studies were categorised as comparative studies or cohort studies. For each article, the following data were collected: year of publication, number of patients, design of the study, aim of the study, patient inclusion criteria, technical and pharmacological aspects of (H)IPEC, timing of (H)IPEC, dosage, type and timing of adjuvant systemic chemotherapy, procedure-related morbidity and mortality of (H)IPEC, follow-up, peritoneal recurrence rate, overall survival, and disease-free survival. A meta-analysis was not intended because of the expected heterogeneity within studies. A descriptive method was used to analyse the available data.

\section{RESULTS}

Out of 1414 search results, 1387 studies were excluded based on title and abstract. Full text of the remaining 27 studies was screened for eligibility. Studies that included patients after CRS for known
PC were excluded, as well as one randomised controlled trial (RCT) in which regional adjuvant treatment consisted of either intraperitoneal or intraportal chemotherapy (Nordlinger et al, 2005). Because the two regional treatment modalities were not analysed as separate groups, the study was excluded from this review. A total of 12 studies reporting on the use of adjuvant (H)IPEC in high-risk CRC patients were identified Figure 1. Studies were categorised as comparative studies $(n=7)$ (Tables 1 and 2) and prospective cohort studies $(n=5)$ (Tables 3 and 4). Among the comparative studies, four RCTs (Sugarbaker et al, 1985; Graf et al, 1994; Scheithauer et al, 1998; Vaillant et al, 2000), two non-randomised comparative studies (Noura et al, 2011; Tentes et al, 2011), and one case-control study were identified (Sammartino et al, 2012).

The oldest of the selected articles was published in 1985 (Sugarbaker et al, 1985), and the most recent publication dated from 2012 (Sammartino et al, 2012). The largest comparative study included 267 patients (Vaillant et al, 2000), and the largest cohort study included 87 patients (Lygidakis et al, 2010). In total, adjuvant (H)IPEC was administered in 463 patients. Five studies confined their inclusion to colon cancer patients only (Kelsen et al, 1994; Scheithauer et al, 1998; Palermo et al, 2000; Vaillant et al, 2000; Seymour et al, 2008; Sammartino et al, 2012), whereas most studies did not exclude rectal cancer patients. One study included only rectal cancer patients (Lygidakis et al, 2010). Of the seven comparative studies, two studies were considered to have a low risk of bias (Graf et al, 1994; Scheithauer et al, 1998), two studies a high risk of bias (Sugarbaker et al, 1985; Tentes et al, 2011), and three studies an intermediate risk of bias (Vaillant et al, 2000; Noura et al, 2011; Sammartino et al, 2012). All cohort studies were considered to have a high risk of bias (Supplementary Information II).

Criteria for the inclusion of patients. Nodal-positive disease and/ or T4 tumours were reported as inclusion criteria in most studies (Sugarbaker et al, 1985; Kelsen et al, 1994; Scheithauer et al, 1998; Palermo et al, 2000; Vaillant et al, 2000; Chouillard et al, 2009; Lygidakis et al, 2010). Three studies included all patients with T3 or T4 tumours, regardless of nodal status (Seymour et al, 2008;

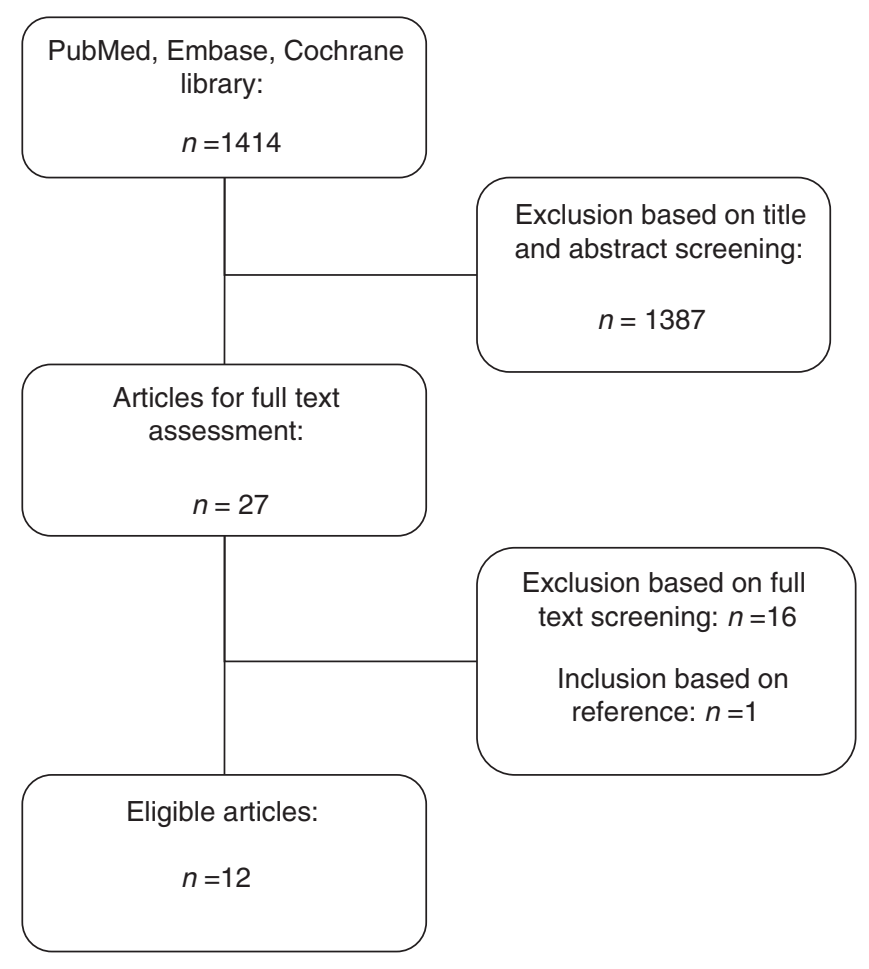

Figure 1. Flow chart of systematic search. 
Table 1. Characteristics of randomised and non-randomised comparative studies

\begin{tabular}{|c|c|c|c|c|c|c|}
\hline $\begin{array}{l}\text { Author, year, } \\
\text { design }\end{array}$ & Inclusion criteria & $\mathbf{n}$ & $\begin{array}{l}\text { Interval } \\
\text { after } \\
\text { resection }\end{array}$ & $\begin{array}{l}\text { Drug, dosage, } \\
\text { administration route }\end{array}$ & $\begin{array}{l}\text { Duration and } \\
\text { frequency }\end{array}$ & $\begin{array}{l}\text { Methods } \\
\text { of i.p. } \\
\text { administration }\end{array}$ \\
\hline $\begin{array}{l}\text { Sugarbaker } \\
\text { et al, } 1985 \\
\text { RCT }\end{array}$ & $\begin{array}{l}\text { Colorectal cancer } \\
\mathrm{N}+, \mathrm{T} 4 \text {, obstruction, } \\
\text { perforation, age }<30 \\
\text { years }\end{array}$ & 30 & 2 months & $\begin{array}{l}\text { i.p. } 5 \mathrm{FU} 1040 \mathrm{mg} \text {; } \\
\text { i.v. } 5 \mathrm{FU} 12 \mathrm{mg} \mathrm{kg}^{-1}\end{array}$ & $\begin{array}{l}\text { Daily for } 5 \text { days every month; } \\
12 \text { cycles } \\
\text { Daily for } 5 \text { days every month; } \\
12 \text { cycles }\end{array}$ & IPC \\
\hline $\begin{array}{l}\text { Graf et al, } \\
1994 \\
\text { RCT }\end{array}$ & $\begin{array}{l}\text { Colorectal cancer } \\
\text { electively operated with } \\
\text { curative intent, exclusion } \\
\text { of stage I }\end{array}$ & 50 & $\begin{array}{l}1 \text { day after surgery } \\
1 \text { day after surgery }\end{array}$ & $\begin{array}{l}\text { i.p. } 5 \mathrm{FU} 500 \mathrm{mg} \mathrm{m}^{-2} \text { per day } \\
\text { and i.v. } \mathrm{LV} 60 \mathrm{mg} \mathrm{m}^{-2} \text { per day } \\
\text { i.p. placebo }\end{array}$ & $\begin{array}{l}4 \mathrm{~h} \text { daily for } 6 \text { consecutive } \\
\text { days } \\
1 \text { cycle } \\
4 \mathrm{~h} \text { daily for } 6 \text { consecutive } \\
\text { days } \\
1 \text { cycle }\end{array}$ & $\begin{array}{l}\text { IPC } \\
\text { IPC }\end{array}$ \\
\hline $\begin{array}{l}\text { Scheithauer } \\
\text { et al, } 1998 \\
\text { RCT }\end{array}$ & $\begin{array}{l}\text { colon cancer } \\
\mathrm{T} 3 / 4 \text { and/or } \mathrm{N}+\end{array}$ & $\begin{array}{l}117 \\
119 \\
\\
119\end{array}$ & $\begin{array}{l}1-5 \text { weeks } \\
1-5 \text { weeks }\end{array}$ & $\begin{array}{l}\text { i.p. LV } 200 \mathrm{mg} \mathrm{m}^{-2}+\text { i.p. } 5 \mathrm{FU} \\
350 \mathrm{mg} \mathrm{m}^{-2} \\
\text { i.v. LV } 200 \mathrm{mg} \mathrm{m}^{-2} \text { and i.v. } \\
\text { 5FU } 350 \mathrm{mg} \mathrm{m}^{-2} \\
\text { p.o. LE } 50 \mathrm{mg} \mathrm{m}^{-2} \text { and i.v. } \\
\text { 5FU } 450 \mathrm{mg} \mathrm{m}^{-2}\end{array}$ & $\begin{array}{l}\text { Day } 1 \text { and } 3 \text { of each i.v. cycle } \\
\text { daily for } 4 \text { consecutive days } \\
\text { every } 4 \text { weeks, total } 6 \text { cycles } \\
\text { Three times daily for } 3 \text { days } \\
\text { every } 2 \text { weeks; } \\
\text { daily for } 5 \text { days, } 2 \text { nd course } \\
\text { after } 4 \text { weeks, weekly } \\
\text { thereafter } \\
\text { total } 6 \text { cycles }\end{array}$ & IPC \\
\hline $\begin{array}{l}\text { Vaillant et al, } \\
2000 \\
\text { RCT }\end{array}$ & $\begin{array}{l}\text { Colon cancer } \\
\text { T3/4 and/or } N+\end{array}$ & $\begin{array}{l}133 \\
134 \\
\end{array}$ & 4-14 days & $\begin{array}{l}\text { i.p. } 5 \mathrm{FU} 600 \mathrm{mg} \mathrm{m}^{-2} \\
\text { i.v. } 1 \mathrm{~g} 5 \mathrm{FU} \\
\text { No chemotherapy }\end{array}$ & $\begin{array}{l}3 \mathrm{~h} \text { daily for } 6 \text { consecutive } \\
\text { days; } \\
1 \text { cycle } \\
\text { Once during surgery }\end{array}$ & IPC \\
\hline $\begin{array}{l}\text { Noura et al, } \\
2011 \\
\text { CS }\end{array}$ & $\begin{array}{l}\text { Colorectal cancer } \\
\text { positive peritoneal } \\
\text { lavage }\end{array}$ & $\begin{array}{l}31^{\mathrm{a}} \\
22\end{array}$ & $\begin{array}{l}\text { Simultaneous } \\
\text { with primary } \\
\text { tumour resection }\end{array}$ & $\begin{array}{l}\text { i.p. } \mathrm{MMC} 20 \mathrm{mg} \\
\mathrm{T}=37^{\circ} \mathrm{C} ; t=1 \mathrm{~h} \\
\text { i.v. } 5 \mathrm{FU} / \mathrm{LV} \text { or } \\
\text { p.o. } 5 \mathrm{FU} \text { derivates }(n=23) \\
\text { i.v. } 5 \mathrm{FU} / \mathrm{LV} \text { orp.o. } 5 \mathrm{FU} \\
\text { derivates }(n=19)\end{array}$ & $\begin{array}{l}\text { Once during surgery } \\
\text { Schedule not specified } \\
\text { Schedule not specified }\end{array}$ & $\begin{array}{l}\text { Closed IPEC } \\
\text { procedure }\end{array}$ \\
\hline $\begin{array}{l}\text { Tentes et al, } \\
2011 \\
\text { CS }\end{array}$ & $\begin{array}{l}\text { Colorectal cancer } \\
\text { T3/4 }\end{array}$ & 西 & $\begin{array}{l}\text { Simultaneous } \\
\text { with primary } \\
\text { tumour resection } \\
1 \text { day }\end{array}$ & $\begin{array}{l}\text { i.p. } \mathrm{MMC} 15 \mathrm{mg} \mathrm{m}^{-2} \\
\mathrm{~T}=42.5-43^{\circ} \mathrm{Ct}=90 \mathrm{minor} \\
\mathrm{O} \times 130 \mathrm{mg} \mathrm{m}^{-2} \\
T=42.5-43^{\circ} \mathrm{C} t=60 \mathrm{~min} \\
\text { i.v. } 5 \mathrm{FU} / \mathrm{LV} \text { in Stage III/IV } \\
\text { i.p. } 5 \mathrm{FU} 600 \mathrm{mg} \mathrm{m}^{-2} \\
\text { i.v. } 5 \mathrm{FU} / \mathrm{LV} \text { in } \\
\text { Stage III/IV) }\end{array}$ & $\begin{array}{l}23 \text { h daily for } 5 \text { consecutive } \\
\text { days; } \\
1 \text { cycle } \\
6 \text { cycles }\end{array}$ & $\begin{array}{l}\text { Open HIPEC } \\
\text { procedure } \\
\text { IPC }\end{array}$ \\
\hline $\begin{array}{l}\text { Sammartino et al, } \\
2012 \\
\text { matched CS }\end{array}$ & $\begin{array}{l}\text { Colon cancer } \\
\text { T3/4NxMO, perforation } \\
\text { (regardless of tumour } \\
\text { stage), signet cell or } \\
\text { mucinous } \\
\text { tumours }\end{array}$ & 25 & $\begin{array}{l}\text { Simultaneous with } \\
\text { primary tumour } \\
\text { resection (with } \\
\text { appendectomy, } \\
\text { omentectomy, resection } \\
\text { of the round hepatic } \\
\text { ligament, and bilateral } \\
\text { ovariectomy) }\end{array}$ & $\begin{array}{l}\text { i.p. } \mathrm{Ox} 460 \mathrm{mg} \mathrm{m}^{-2} \\
\mathrm{~T}=43^{\circ} \mathrm{C} t=30 \mathrm{~min}^{-} \\
\text {i.v. } 5 \mathrm{FU} 400 \mathrm{mg} \mathrm{m}^{-2}+\mathrm{LV} \\
20 \mathrm{mg} \mathrm{m}^{-2} \\
\text { i.v. } 5 \mathrm{FU} / \mathrm{Ox}(n=13) \\
\text { i.v. } 5 \mathrm{FU} / \mathrm{Ox}(n=23)\end{array}$ & $\begin{array}{l}\text { Once during surgery } \\
\text { not specified } \\
\text { Not specified }\end{array}$ & $\begin{array}{l}\text { Open HIPEC } \\
\text { procedure }\end{array}$ \\
\hline
\end{tabular}

Abbreviations: $\mathrm{CS}=$ non-randomised comparitive study; $5 \mathrm{FU}=$ fluorouracil; $(\mathrm{H}) \mathrm{IPEC}=$ (hyperthermic) intraperitoneal chemotherapy; i.p. $=$ intraperitoneal; $I \mathrm{PC}=$ intraperitoneal catheter; i.v. = intravenous; $L E=$ levamisole; $L V=$ leucovorin; $M M C=$ mitomycine-C; Ox=oxaliplatin; p.o. =oral; $R C T=$ randomised controlled trial; $T=$ temperature of intraperitoneal infusion; $t=$ duration of infusion.

${ }^{a}$ Selection based on general patient status and invasiveness of surgery. Intraperitoneal catheter simultaneously placed with primary tumour resection or via a percutaneous approach, with or without a subcutaneous reservoir. 
Table 2. End points in comparative studies

\begin{tabular}{|c|c|c|c|c|c|c|}
\hline Author & Group & $\begin{array}{c}\text { Overall/disease-free } \\
\text { survival }\end{array}$ & $\begin{array}{l}\text { Peritoneal } \\
\text { recurrence } \\
\text { rate }\end{array}$ & Complications & $\begin{array}{l}\text { Treatment- } \\
\text { related } \\
\text { mortality }\end{array}$ & Tolerance \\
\hline $\begin{array}{l}\text { Sugarbaker } \\
\text { et al, } 1985\end{array}$ & $\begin{array}{l}\text { i.p. 5FU } \\
\text { i.v. 5FU } \\
P \text {-value }\end{array}$ & $\begin{array}{c}\text { Median overall survival } \\
\text { (months) } \\
46.3 \\
47.5 \\
\\
\mathrm{NS}^{\mathrm{a}}\end{array}$ & $\begin{array}{l}20 \% \\
91 \% \\
0.003^{b}\end{array}$ & $\begin{array}{l}\text { Serious complications (n) } \\
\qquad \begin{array}{l}15 \\
16 \\
\mathrm{nr}\end{array}\end{array}$ & $\mathrm{nr}$ & $\begin{array}{l}\text { Dose-limiting events } \\
\text { Mucositis } 25 \% \text {, leucocyte } \\
\text { suppression } 60 \% \text {, } \\
\text { abdominal discomfort } \\
\text { abdominal pain } \\
\text { Mucositis } 40 \% \text {, leucocyte } \\
\text { suppression } 20 \% \text {, } \\
\text { abdominal discomfort } \\
\text { abdominal pain } \\
\text { nr }\end{array}$ \\
\hline $\begin{array}{l}\text { Graf et al, } \\
1994\end{array}$ & $\begin{array}{l}\text { i.p. } 5 \mathrm{FU}+\text { i.v. } \\
\mathrm{LV} \\
\text { i.p. placebo } \\
\text { P-value }\end{array}$ & $\mathrm{nr}$ & $\mathrm{nr}$ & $\begin{array}{c}\text { Post-operative } \\
\text { complications (n) } \\
11\end{array}$ & $\begin{array}{c}\text { In hospital or } \\
\text { within } 30 \text { days (n) } \\
0 \\
0 \\
\mathrm{nr}\end{array}$ & $\begin{array}{l}\text { Tolerance } \\
\text { Nausea } 5 \% \text {, diarrhoea } 2 \% \text {, } \\
\text { allergic reaction } 2 \% \text {, } \\
\text { infusion-connected pain } \\
\text { Nausea } 15 \% \text {, diarrhoea } 7 \% \text {, } \\
\text { allergic reaction } 0 \% \text {, } \\
\text { infusion-connected pain } \\
\text { nr }\end{array}$ \\
\hline $\begin{array}{l}\text { Scheithauer } \\
\text { et al, } 1998\end{array}$ & $\begin{array}{l}\text { i.p. +i.v. 5FU/ } \\
\text { LV } \\
\text { i.v. } 5 F U+\text { p.o. } \\
\text { LE } \\
\text { P-value }\end{array}$ & $\begin{array}{c}\text { Actuarial 4-year survival } \\
\text { rate } \\
83 \% \\
65 \% \\
\\
\mathrm{nr}\end{array}$ & $\begin{array}{c}4 \text { years } \\
8 \% \\
21 \% \\
0.005^{c}\end{array}$ & $\begin{array}{c}\begin{array}{c}\text { Life-threateningside } \\
\text { effects }(n)\end{array} \\
0 \\
0 \\
\mathrm{nr}\end{array}$ & $\begin{array}{c}\text { Treatment- } \\
\text { related death (n) } \\
0 \\
0 \\
n r\end{array}$ & $\begin{array}{l}\text { Severe treatment- } \\
\text { associated side effects } \\
13 \% \\
3 \% \\
0.01\end{array}$ \\
\hline $\begin{array}{l}\text { Vaillant et al, } \\
2000\end{array}$ & $\begin{array}{l}\text { i.p. 5FU } \\
\text { no CT } \\
P \text {-value }\end{array}$ & 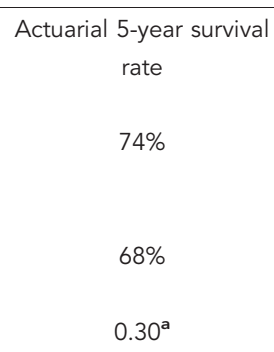 & $\begin{array}{c}8 \% \\
10 \% \\
\mathrm{nr}\end{array}$ & $\begin{array}{c}\text { Post-operative } \\
\text { complications (n) } \\
26 \\
16 \\
\mathrm{nr}\end{array}$ & $\begin{array}{c}\text { Post-operative } \\
\text { mortality (n) } \\
2 \\
0 \\
\text { nr }\end{array}$ & $\begin{array}{l}\text { Fair } 14.9 \% \\
\text { poor } 3.3 \% \\
\text { nr }\end{array}$ \\
\hline $\begin{array}{l}\text { Noura et al, } \\
2011\end{array}$ & $\begin{array}{l}\text { i.p. MMC } \\
+ \text { (i.v. 5FU/ } \\
\text { Ox) } \\
\text { (i.v. 5FU/Ox) } \\
\text { P-value }\end{array}$ & $\begin{array}{c}\text { Actuarial } 5 \text {-year cancer- } \\
\text { specific survival rate } \\
54.3 \% \\
\\
9.5 \% \\
0.0001^{\mathrm{a}}\end{array}$ & $\begin{array}{c}\text { Actuarial 5-year } \\
12 \% \\
59.9 \% \\
0.0003^{\mathrm{a}}\end{array}$ & $\begin{array}{c}\text { IPEC-related post- } \\
\text { operative complications } \\
(\mathrm{n}) \\
\text { Grade III/IV }=1 \\
\text { Grade III/IV }=0 \\
\mathrm{nr}\end{array}$ & $\begin{array}{c}0 \\
0 \\
\mathrm{nr}\end{array}$ & $\begin{array}{l}\text { Grade } 3 \text { complications } \\
\text { related to i.p. lavage }\end{array}$ \\
\hline
\end{tabular}




\begin{tabular}{|c|c|c|c|c|c|c|}
\hline Author & Group & $\begin{array}{c}\text { Overall/disease-free } \\
\text { survival }\end{array}$ & $\begin{array}{l}\text { Peritoneal } \\
\text { recurrence } \\
\text { rate }\end{array}$ & Complications & $\begin{array}{l}\text { Treatment- } \\
\text { related } \\
\text { mortality }\end{array}$ & Tolerance \\
\hline \multirow[t]{4}{*}{$\begin{array}{l}\text { Tentes et al, } \\
2011\end{array}$} & & $\begin{array}{c}\text { Actuarial 3-year survival } \\
\text { rate }\end{array}$ & $\mathrm{nr}$ & Overall complications ( $n$ ) & $\begin{array}{l}\text { Hospital mortality } \\
\text { (n) }\end{array}$ & Tolerance \\
\hline & $\begin{array}{l}\text { HIPEC, MMC } \\
\text { or Ox } \\
\text { (i.v. } 5 F U / L V \text { ) }\end{array}$ & $100 \%$ & $\mathrm{nr}$ & 16 & 1 & $\mathrm{nr}$ \\
\hline & $\begin{array}{l}\text { i.p. 5FU } \\
\text { (i.v. } 5 F U / L V)\end{array}$ & $69 \%$ & $\mathrm{nr}$ & & 9 & $\mathrm{nr}$ \\
\hline & $P$-value & $0.011^{a}$ & $\mathrm{nr}$ & 0.05 & 0.009 & $\mathrm{nr}$ \\
\hline \multirow[t]{4}{*}{$\begin{array}{l}\text { Sammartino } \\
\text { et al, } 2012\end{array}$} & & $\begin{array}{l}\text { Median disease-free } \\
\text { survival (months) }\end{array}$ & & Grades I-IV (n) & & $\begin{array}{l}\text { HIPEC toxicity } \\
\text { Grade II }\end{array}$ \\
\hline & $\begin{array}{l}\text { HIPEC Ox } \\
\text { (i.v. } 5 F U / O x \text { ) }\end{array}$ & 36.8 & $4 \%$ & $\begin{array}{l}\text { Grade } I / I I=3 \\
\text { Grade } I I=0 \\
\text { Grade } I V=1\end{array}$ & $\mathrm{nr}$ & 0 \\
\hline & (i.v. 5FU/Ox) & 21.9 & $22 \%$ & $\begin{array}{l}\text { Grade } I / I I=5 \\
\text { Grade } I I I=1 \\
\text { Grade } I V=3\end{array}$ & $\mathrm{nr}$ & \\
\hline & $P$-value & $<0.01^{\mathrm{a}}$ & $<0.05^{b}$ & & $\mathrm{nr}$ & $\mathrm{nr}$ \\
\hline
\end{tabular}

Tentes et al, 2011; Sammartino et al, 2012). One study included all electively operated patients, with exclusion of patients with stage I disease (Graf et al, 1994). Additional inclusion criteria were malignant obstruction or perforation (Sugarbaker et al, 1985; Kelsen et al, 1994; Chouillard et al, 2009; Sammartino et al, 2012), positive peritoneal lavage (Chouillard et al, 2009; Noura et al, 2011), CRC under the age of 30 years (Sugarbaker et al, 1985), and signet cell or mucinous tumours (Sammartino et al, 2012). In three studies using simultaneous (H)IPEC, eligibility of the patients had to be determined before or during initial tumour resection. Selection was based on intra-operative cytology of peritoneal lavage in the study by Noura et al (2009). Tumour depth (pT stage) and specific histological features (signet cell or mucinous adenocarcinoma) were assessed intra-operatively in the study bySammartino et al (2012). Tentes et al (2011) used clinical T3 or T4 stage as inclusion criterion; a few patients underwent (H)IPEC for a pathological T2N0 stage, who were excluded from analysis afterwards.

Technique, frequency and timing of (H)IPEC. The intraperitoneal treatment schedules are summarised in Tables 1 and 3 and can be divided into two main approaches. Initial studies have used peritoneal catheters with or without a subcutaneous reservoir to treat patients in an ambulatory setting (seven studies). Later studies applied (H)IPEC in an intra-operative setting with either a completely open approach, an open approach with closed perfusion, or a laparoscopic approach, with or without hyperthermia (five studies).

The way of administration was linked to the type of chemotherapeutic agent. In all studies (Sugarbaker et al, 1985;
Graf et al, 1994; Kelsen et al, 1994; Scheithauer et al, 1998; Palermo et al, 2000; Vaillant et al, 2000; Seymour et al, 2008) or study arms (Tentes et al, 2011) with fluoropyrimidine-based therapy, repetitive sessions of peritoneal chemotherapy administration were performed through peritoneal catheters (repeated ambulatory IPEC). In three studies, patients received a single cycle of daily lavages for several days (Graf et al, 1994; Vaillant et al, 2000; Tentes et al, 2011), but most studies applied multiple (monthly) treatment cycles. Treatment with repeated ambulatory IPEC started the day after surgery in two studies (Graf et al, 1994; Tentes et al, 2011), but was mostly delayed to several days up to 2 months after primary tumour resection. Catheters were placed intra-abdominal during initial tumour resection or inserted percutaneous at a later stage. Catheters were left in situ during the entire treatment program, unless removal was indicated by port-site infection or obstruction of the catheter.

Intra-operative (H)IPEC was used in five studies, all using mitomycin-C (MMC) and/or oxaliplatin (Chouillard et al, 2009; Lygidakis et al, 2010; Noura et al, 2011; Tentes et al, 2011; Sammartino et al, 2012). These procedures were single stage, except for the study by Lygidakis et al (2010) in which a second and a third lavage were scheduled after 28 days and 2 years, respectively. Intra-operative $(\mathrm{H}) \mathrm{IPEC}$ procedures were performed during initial tumour resection in three studies, either before or directly after reconstruction of the continuity of the alimentary tract. Simultaneous HIPEC was performed by the completely open HIPEC technique in two studies (Tentes et al, 2011; Sammartino et al, 2012) and by closed perfusion without hyperthermia in a third study (Noura et al, 2011). In one of these studies, resection of 


\begin{tabular}{|c|c|c|c|c|c|c|}
\hline $\begin{array}{l}\text { Author, } \\
\text { year }\end{array}$ & Inclusion criteria & $n$ & $\begin{array}{l}\text { Interval } \\
\text { after } \\
\text { resection }\end{array}$ & $\begin{array}{l}\text { Drug, dosage, } \\
\text { administration route }\end{array}$ & Duration/frequency & $\begin{array}{l}\text { Methods } \\
\text { of i.p. } \\
\text { administration }\end{array}$ \\
\hline $\begin{array}{l}\text { Kelsen } \\
\text { et al, } 1994\end{array}$ & $\begin{array}{l}\text { Colon cancer } \\
\mathrm{N}+; \mathrm{T} 4 \text { with } \\
\text { obstruction or } \\
\text { perforation, or } \\
\text { resected } \\
\text { intra-abdominal M1 }\end{array}$ & 26 & $\begin{array}{l}2-5 \text { days after } \\
\text { resection }\end{array}$ & $\begin{array}{l}\text { i.p. floxuridine } 500 \mathrm{mg} \mathrm{m}^{-2} \\
\text { i.p. LV } 120 \mathrm{mg} \mathrm{m}^{-2} \\
\text { p.o. levamisol } 50 \mathrm{mg} \\
\text { i.v. } 5 \text { FU bolus } 200-450 \mathrm{mg} \mathrm{m}^{-2} \\
\text { dose escalation } \\
\text { i.v. } 5 \text { FU } 450 \mathrm{mg} \mathrm{m}^{-2} \text { per week }\end{array}$ & $\begin{array}{l}\text { Twice daily for } 3 \text { consecutive days } \\
\text { every } 2 \text { weeks } \\
3 \text { cycles } \\
3 \text { times a day for } 3 \text { days every } 2 \\
\text { weeks, total } 1 \text { year } \\
\text { During } 3 \text { rd i.p. cycle, daily for } 5 \text { days } \\
\text { Every week, total } 1 \text { year }\end{array}$ & IPC \\
\hline $\begin{array}{l}\text { Palermo } \\
\text { et al, } 2000\end{array}$ & $\begin{array}{l}\text { Colon cancer } \\
\mathrm{T} 3 / 4, \mathrm{~N}+\end{array}$ & 45 & $\mathrm{nr}$ & $\begin{array}{l}\text { i.p. } 5 \mathrm{FU} 20 \mathrm{mg} \mathrm{kg}^{-1} \\
\text { radiotherapy } 150 \mathrm{cGy}\end{array}$ & $\begin{array}{l}\text { Daily } 60-90 \text { min for } 5 \text { consecutive } \\
\text { days every } 4 \text { weeks } \\
6 \text { cycles } \\
\text { Daily for three weeks, } 2 \text { cycles with } \\
1 \text { week interval }\end{array}$ & IPC \\
\hline $\begin{array}{l}\text { Seymour } \\
\text { et al, } 2008\end{array}$ & $\begin{array}{l}\text { Colon cancer } \\
\text { T4 }\end{array}$ & $47^{a}$ & $\begin{array}{l}\text { Median } 57 \\
\text { days } \\
(15-148)\end{array}$ & $\begin{array}{l}\text { i.p. } 5 F U 400 \mathrm{mg} \mathrm{m}^{-2} \\
\text { i.p. } L V 20 \mathrm{mg} \mathrm{m}^{-2} \\
\text { i.v. } 5 F U / L V\end{array}$ & $\begin{array}{l}\text { Separate cohorts with frequencies of } \\
\text { once per } 4,3,2 \text {, or } 1 \text { week(s) } \\
\text { total } 24 \text { weeks } \\
\text { Once every week, max } 24 \text { weeks. }\end{array}$ & IPC \\
\hline $\begin{array}{l}\text { Chouillard } \\
\text { et al, } 2009\end{array}$ & $\begin{array}{l}\text { Colorectal cancer } \\
\text { T4, pN2, perforation } \\
\text { or obstruction } \\
\text { (regardless of } \\
\text { tumour stage); positive } \\
\text { peritoneal lavage }\end{array}$ & 16 & 5-8weeks & $\begin{array}{l}\text { i.p. } \mathrm{MMC} 80 \mathrm{mg} \mathrm{m}^{-2}: \mathrm{T}=42- \\
44^{\circ} \mathrm{C} ; t=35-45 \mathrm{~min} \\
\text { adjuvant i.v. treatment not } \\
\text { mentioned }\end{array}$ & Once & $\begin{array}{l}\text { Laparoscopic } \\
\text { HIPEC } \\
\text { procedure }\end{array}$ \\
\hline $\begin{array}{l}\text { Lygidakis } \\
\text { et al, } 2010\end{array}$ & $\begin{array}{l}\text { Rectal cancer } \\
\mathrm{N}+\text {, neurovascular } \\
\text { involvement }\end{array}$ & 87 & 22 days & $\begin{array}{l}\text { i.p. } 5 F U, O x, L V \text {, Irinotecan } \\
T=43^{\circ} \mathrm{C} ; t=60 \mathrm{~min} \\
\text { i.v. } 5 \mathrm{FU}, \mathrm{Ox}, \mathrm{LV} \text {, Irinotecan }\end{array}$ & $\begin{array}{l}\text { Second lavage after } 25 \text { days and third } \\
\text { lavage after } 2 \text { years. } \\
\text { Every month, } 4 \text { cycles }\end{array}$ & $\begin{array}{l}\text { Laparoscopic } \\
\text { HIPEC } \\
\text { procedure }\end{array}$ \\
\hline \multicolumn{7}{|c|}{$\begin{array}{l}\text { Abbreviations: } \mathrm{Cis}=\text { cisplatinum; } 5 \mathrm{FU}=\text { fluorouracil; } \mathrm{HIPEC}=\text { hyperthermic intraperitoneal chemotherapy; i.p. }=\text { intraperitoneal; } I \mathrm{PC}=\text { intraperitoneal } \text { catheter; } \mathrm{i} . \mathrm{v} .=\text { intravenous; } \mathrm{LV}= \\
\text { leucovorin; } \mathrm{MMC}=\text { mitomycine- } \mathrm{C} ; \mathrm{Ox}=\text { oxaliplatin; } \mathrm{p} . \mathrm{o} .=\text { oral; } \mathrm{t}=\text { duration of infusion; } \mathrm{T}=\text { temperature of intraperitoneal infusion. } \\
\mathrm{a}_{10} \text { out of } 12 \text { colorectal cancer histology in pharmacokinetic study and } 37 \text { out of } 44 \text { colorectal cancer histology in frequency-escalation study. }\end{array}$} \\
\hline
\end{tabular}

the primary tumour was extended with an appendectomy, omentectomy, resection of the round hepatic ligament, and bilateral ovariectomy in women using a completely open HIPEC technique (Sammartino et al, 2012). Early post-operative HIPEC using a laparoscopic approach was used in the two remaining studies at 3-8 weeks from primary tumour resection (Chouillard et al, 2009; Lygidakis et al, 2010).

(H)IPEC agents and combinations with systemic treatment. $5 \mathrm{FU}$ was used as a single chemotherapeutic agent for intraperitoneal use in five studies (Sugarbaker et al, 1985; Graf et al, 1994; Palermo et al, 2000; Vaillant et al, 2000; Tentes et al, 2011). Intraperitoneal LV was used in combination with a fluoropyrimidine analogue (e.g., 5FU or floxuridine) in three studies (Kelsen et al, 1994; Scheithauer et al, 1998; Seymour et al, 2008), a combination of intraperitoneal 5FU and intravenous LV was used in one study (Graf et al, 1994), and three studies used concurrent intraperitoneal and intravenous 5FU administration either combined with intravenous LV or oral levamisole (Kelsen et al, 1994; Scheithauer et al, 1998; Seymour et al, 2008). MMC was exclusively used during (H)IPEC in two studies (Chouillard et al, 2009; Noura et al, 2011), either MMC or oxaliplatin in one study (Tentes et al, 2011), and exclusively oxaliplatin in another study (Sammartino et al, 2012). Lygidakis et al (2010) described the use of 5FU, oxaliplatin, LV, irinotecan, and MMC, but chemotherapy schedules were not further specified. Hyperthermia was used to potentiate the effect of MMC or oxaliplatin in all studies, except for the study by Noura et al (2011). The effect of intraperitoneal oxaliplatin was further potentiated by the intravenous administration of 5FU/LV just before the start of the $(\mathrm{H})$ IPEC procedure.

Fluoropyrimidine- or oxaliplatin-based adjuvant systemic treatment was given in addition to adjuvant (H)IPEC in seven studies (Tables 1 and 3). (H)IPEC was not followed by adjuvant systemic therapy in four studies (Sugarbaker et al, 1985; Graf et al, 1994; Palermo et al, 2000; Vaillant et al, 2000). In one of these studies, a combined adjuvant treatment schedule was used of repeated ambulatory intraperitoneal 5FU/LV administration and low doses of radiotherapy (Palermo et al, 2000). In one additional study, the use of systemic treatment after adjuvant HIPEC was not mentioned (Chouillard et al, 2009).

Tolerance, morbidity, and mortality of (H)IPEC. The tolerance, morbidity, and mortality for the different strategies are summarised in Tables 2 and 4. Intolerance of intraperitoneal treatment was reported as dose-limiting events, failure to complete the treatment, severe treatment-associated side effects, and grading of (H)IPEC toxicity. Various grading systems or definitions, such as post-operative complications, serious complications, and lifethreatening side effects were used for the description of morbidity.

Mortality was defined as treatment-related mortality, in-hospital mortality, or mortality within 30 days.

Complications such as infection (Seymour et al, 2008), chemical peritonitis (Sugarbaker et al, 1985; Palermo et al, 2000), diabetes (Sugarbaker et al, 1985), bowel perforation (Seymour et al, 2008), abdominal discomfort and pain (Sugarbaker et al, 1985; 


\begin{tabular}{|c|c|c|c|c|c|}
\hline Author & Overall survival & $\begin{array}{l}\text { Peritoneal disease } \\
\text { recurrence }\end{array}$ & Complications & $\begin{array}{l}\text { Treatment- } \\
\text { related } \\
\text { mortality }\end{array}$ & Tolerance \\
\hline $\begin{array}{l}\text { Kelsen } \\
\text { et al, } 1994\end{array}$ & $\mathrm{nr}$ & $\mathrm{nr}$ & $\begin{array}{l}\text { No increase in post-operative } \\
\text { morbidity } \\
1 \text { catheter removed of peritonitis }\end{array}$ & $0 \%$ & $\begin{array}{l}\text { i.p.: Gr3 + } \\
\text { myelosuppression 2/26 } \\
\text { i.v. } 5 F U 300 \text { mg: Gr3 }+ \\
\text { myelosuppression } 2 / 7 \text {, } \\
\text { Gr3 + mucositis } 4 / 7 \\
\text { i.v. } 5 \mathrm{FU} 400 \mathrm{mg} \text { : Gr3 + } \\
\text { myelosuppression } 2 / 3 \text {, } \\
\text { Gr3 + mucositis } 2 / 3\end{array}$ \\
\hline $\begin{array}{l}\text { Palermo } \\
\text { et al, } 2000\end{array}$ & $\begin{array}{l}\text { Median follow-up } \\
130 \text { months (108-163) } \\
5 \text {-year DFS 51\% } \\
5 \text {-year OS 56\% }\end{array}$ & $\begin{array}{l}5 / 45 \text { ( } n=4 \\
\text { relaparotomy because } \\
\text { of small bowel } \\
\text { obstruction) }\end{array}$ & $\begin{array}{l}\text { Gr3 chemical peritonitis } 7 / 45 \\
\text { patients, no bacterial peritonitis } \\
\text { Gr3 nausea/vomiting } 3 / 45 \\
\text { Gr4 nausea/vomiting } 1 / 45 \\
\text { Gr3 haematologic toxicity } 2 / 45 \\
\text { Small bowel obstruction } 6 / 45\end{array}$ & $0 \%$ & $\begin{array}{l}\text { Grade I toxicity }=49 \% \\
\text { Grade II toxicity }=19 \% \\
\text { Grade III toxicity }=15 \% \\
\text { Grade IV toxicity }=2 \%\end{array}$ \\
\hline $\begin{array}{l}\text { Seymour } \\
\text { et al, } 2008\end{array}$ & $\mathrm{nr}$ & $\mathrm{nr}$ & $\begin{array}{l}\text { Failure of intraperitoneal access } \\
n=10 \text { : initial failure } 7 \text { (pain } n=3 \text {; } \\
\text { leakage } n=2 \text {; infection } n=1 \text {; bowel } \\
\text { perforation } n=1 \text { ) secondary failure } 3 \\
\text { (leakage } n=1 \text {; blockage } n=2 \text { ) } \\
\text { reason for stopping i.p. treatment: } \\
\text { mostly abdominal pain }\end{array}$ & $\mathrm{nr}$ & $\begin{array}{l}\text { Tolerance per treatment } \\
\text { frequency } \\
\text { i.p. per } 4 \text { weeks } \mathrm{nr} \\
\text { i.p. per } 3 \text { weeks } 87 \% \\
\text { i.p. per } 2 \text { weeks } 77 \% \\
\text { i.p. per week } 0 \%\end{array}$ \\
\hline $\begin{array}{l}\text { Chouillard } \\
\text { et al, } 2009\end{array}$ & $\begin{array}{l}\text { Median follow-up } 15.5 \text { months } \\
2 / 16 \text { colorectal cancer patients } \\
\text { died at the end of FU. } 3 / 14 \text { alive } \\
\text { with distant metastasis }\end{array}$ & $\begin{array}{l}\text { Peritoneal recurrence } \\
\text { rate } 0 \%\end{array}$ & $\begin{array}{l}\text { Overall } n=21 \\
\text { Major } n=4 \\
\text { Minor } n=11\end{array}$ & $0 \%$ & $\begin{array}{l}\text { Major: } \\
\text { bone marrow aplasia } \\
n=2 \\
\text { Minor: } \\
\text { low platelet count } n=11 \text {, } \\
\text { leucopenia } n=9 \text {, fatigue } \\
n=8 \text {, nausea } n=7\end{array}$ \\
\hline $\begin{array}{l}\text { Lygidakis } \\
\text { et al, } 2010\end{array}$ & 1-year OS 100\% & $\begin{array}{l}\text { 2-year peritoneal } \\
\text { recurrence rate } 3 \%\end{array}$ & 0 & 0 & $\begin{array}{l}\text { No drug toxicity during } \\
\text { hospital stay }\end{array}$ \\
\hline
\end{tabular}

Scheithauer et al, 1998; Vaillant et al, 2000), intestinal obstruction or ileus (Graf et al, 1994), and intestinal polyposis (Sugarbaker et al, 1985) were specifically associated with repeated IPEC via intraperitoneal catheters. Seymour et al (2008) studied the morbidity of repetitive intraperitoneal treatment with 5FU/LV by escalating the treatment frequency. The authors found that a weekly frequency was not tolerated, mostly due to abdominal pain (Seymour et al, 2008). Specific complications related to operative (H)IPEC were catheter or port-site problems (such as cellulitis) (Chouillard et al, 2009; Noura et al, 2011), pancreatitis (Tentes et al, 2011; Sammartino et al, 2012), and haematological toxicity with bone marrow aplasia in two patients from one study (Chouillard et al, 2009). Anastomotic failure was not associated with (H)IPEC in any of the studies.

Scheithauer et al (1998) reported a severe adverse reaction rate of $13 \%$ in patients that received repeated IPEC with 5FU/LV compared with $3 \%$ in patients that did not receive intraperitoneal treatment. This was the only study in which (H)IPEC showed a significantly higher rate of treatment-related side effects compared with controls. However, the authors concluded that treatmentassociated side effects were infrequent and generally mild.

Graf et al (1994) compared repeated IPEC using 5FU with repeated IPEC using a placebo. Two patients in the 5FU group were diagnosed with localised peritonitis $v s$ no patients in the placebo group. However, intestinal obstruction requiring surgery and wound infection occurred more often in the placebo group
(1 vs 2 and 4 vs 9, respectively). The overall rate of post-operative complications was not significantly different between the 5FU and placebo groups.

In the study of Tentes et al (2011), HIPEC with MMC or oxaliplatin simultaneously with the primary resection was compared with early post-operative-repeated IPEC using 5FU via a peritoneal catheter. Overall complications occurred significantly more often in the repeated IPEC group. In addition, a significant difference in hospital mortality rate was reported with 9 out of 67 (13\%) deaths in the repeated ambulatory IPEC group compared with 1 out of 40 (3\%) in the intra-operative HIPEC group. Causes of death were not provided.

Survival and incidence of PC in comparative studies. Oncological outcome parameters were analysed in six out of seven comparative studies (Table 2; Sugarbaker et al, 1985; Scheithauer et al, 1998; Vaillant et al, 2000; Noura et al, 2011; Tentes et al, 2011; Sammartino et al, 2012). All three survival studies on operative (H)IPEC reported a significant impact on either overall or disease-free survival (Noura et al, 2011; Tentes et al, 2011; Sammartino et al, 2012). An absolute difference in 5-year cancerspecific survival of $44.8 \%$ was found by Noura et al (2011). Tentes et al (2011) reported a significant difference in 3-year overall survival rate of $31.0 \%$, in favour of the operative (H)IPEC group compared with the IPEC group (100 vs 69\%). Sammartino et al (2012) showed a difference in median disease-free survival of 14.9 
months in favour of the operative (H)IPEC group (36.8 vs 21.9 months). From the studies that compare repeated ambulatory IPEC with no intraperitoneal chemotherapy, Vaillant et al (2000) and Scheithauer et al (1998) described absolute differences in overall survival rates of $6.0 \%$ and $18.0 \%$, respectively, in favour of the IPEC groups, but these were not tested for significance. The study by Sugarbaker et al (1985) revealed no significant difference in overall survival.

Five studies compared the peritoneal recurrence rate after (H)IPEC with a control group (Sugarbaker et al, 1985; Scheithauer et al, 1998; Vaillant et al, 2000; Noura et al, 2011; Sammartino et al, 2012). Peritoneal recurrence rates varied between 4 and $91 \%$. A significant difference in the incidence of PC in favour of adjuvant (H)IPEC was found in four studies (Table 2; Sugarbaker et al, 1985; Scheithauer et al, 1998; Noura et al, 2011; Sammartino et al, 2012). Vaillant et al (2000) reported a difference in the incidence of PC of $2.0 \%$ between the treatment and control groups but this was not tested for significance.

\section{DISCUSSION}

The effectiveness of modern systemic therapy in treating peritoneal dissemination of CRC in the adjuvant and metastatic setting has been questioned, in contrast to its success in the prevention and treatment of hematogenous spread (Andre et al, 2009; Kopetz et al, 2009). Once peritoneal metastases have developed, natural prognosis is limited to 3-9 months (Chu et al, 1989; Sadeghi et $a l, 2000)$ and patients respond poorly to systemic treatment (Franko et al, 2012; Klaver et al, 2012). CRS/HIPEC improves survival in selected patients with macroscopic PC (Verwaal et al, 2003; Elias et al, 2009), but morbidity and oncological outcome of this multimodality treatment highly depends on tumour load (Verwaal et al, 2004, 2008). Therefore, one could hypothesise that (H)IPEC in an adjuvant setting may reduce the development of PC in high-risk CRC patients.

This systematic review illustrates different approaches of adjuvant (H)IPEC to prevent PC in high-risk CRC patients. Initial studies used fluoropyramidine-based regimens. These studies did not uniformly demonstrate a benefit in overall survival. In addition, repetitive administration of the cytotoxic agent through peritoneal catheters during several weeks up to even 1 year resulted in discomfort and morbidity. From 2009, results are published for MMC and oxaliplatin as intraperitoneal cytotoxic agents in the adjuvant setting. MMC is not typically used in the management of CRC but is frequently used as intraperitoneal chemotherapeutic agent. It was the first chemotherapeutic agent to be studied in heated solution and showed rapid absorption, mostly in local tissue, resulting in high drug exposure to the peritoneal surface (Panteix et al, 1993). Intraperitoneal MMC has been used successfully as intraperitoneal chemotherapy since the 1980s for a variety of malignancies, both as adjuvant therapy (Yu et al, 1998) or in combination with CRS (Verwaal et al, 2003). Experimental studies on intraperitoneal use of oxaliplatin started in the 1990s (Los et al, 1990), and the first clinical studies with intraperitoneal oxaliplatin were published at the beginning of the 21 st century (Elias et al, 2002). MMC and oxaliplatin are non-cell-cycle dependent, which is the reason why intraperitoneal chemotherapy administration could be reduced to a single procedure.

Single HIPEC procedure under general anaesthesia is safe and potentially associated with less morbidity and less discomfort for the patient than repetitive ambulatory administration of intraperitoneal chemotherapy. The only case of mortality after intraoperative (H)IPEC was reported in the study by Tentes et al (2011). Furthermore, intra-operative (H)IPEC is less likely to interfere with adjuvant systemic treatment. (H)IPEC under general anaesthesia enables the use of hyperthermia and guarantees uniform distribution of the cytotoxic agent throughout the entire abdominal cavity. Considering tissue penetration of intraperitoneal chemotherapy, a laparoscopic approach has been suggested to be superior to an open approach because of increased intra-abdominal pressure in an animal study (Gesson-Paute et al, 2008).

Adjuvant intra-operative (H)IPEC with MMC or oxaliplatin followed by adjuvant systemic therapy was suggested to reduce peritoneal recurrence and improve survival compared with control patients who underwent adjuvant systemic therapy alone in the included studies. Nonetheless, the quality of the currently available data does not allow for a definitive conclusion on the effectiveness of adjuvant (H)IPEC in high-risk CRC patients. The included studies have several limitations. No standardised outcome assessments were used. Studies were relatively underpowered and end points were often chosen differently among the included studies. The nonrandomised comparative studies have a substantial risk of bias. All these methodological shortcomings hamper interpretation of the currently available evidence on outcome of adjuvant (H)IPEC.

One of the most important issues in future research on adjuvant (H)IPEC is the selection of patients. The included studies of the present review used varying definitions for high-risk patients. Differences in patient selection may have attributed to the observed variance of effect of adjuvant (H)IPEC within the different studies. In a recent systematic review by Honore et al (2013), perforation, local peritoneal nodules present at primary resection, and isolated ovarian metastasis are considered to be associated with the highest risk of PC development. T4(a) stage, mucinous or signet ring cell histology, positive peritoneal lavage, and obstruction are reported as intermediate risk factors (Jayne et al, 2002; Yang et al, 2004; Rekhraj et al, 2008; Catalano et al, 2009; Noura et al, 2009; Elias et al, 2011; Hompes et al, 2012; Honore et al, 2013). Risk assessment studies and possibly biomarker studies are needed to further define selection criteria.

The second most important issue to address is the most optimal timing of adjuvant (H)IPEC. (H)IPEC can be performed simultaneous with primary tumour resection, within days, weeks or months after primary resection, or following adjuvant systemic treatment. Even a compulsory waiting period of 6 months after adjuvant systemic treatment has been suggested. The best option is still unclear (Chouillard et al, 2009; Elias et al, 2011; Sammartino et al, 2012). Timing should probably be tailored to the patients' clinical situation and risk profile. If a patient fulfils the criteria of a high-risk patient before primary resection, a simultaneous HIPEC can be planned. For patients with uncomplicated surgery and intra-operative diagnosis of high-risk features, an early postoperative procedure would probably be most suitable as morbidity is limited. In patients with complicated surgery or a complicated primary tumour (i.e., perforation with or without sepsis, or obstruction), adjuvant HIPEC should be delayed or may be reconsidered after completing adjuvant systemic chemotherapy.

In conclusion, adjuvant (H)IPEC possibly leads to a reduction of metachronous $\mathrm{PC}$ in patients undergoing curative resection of high-risk CRC. Current evidence suggests that adjuvant HIPEC after primary tumour resection is feasible and well tolerated. Welldesigned RCTs are warranted to evaluate the impact on oncological outcome, the effects on the quality of life, and the cost effectiveness of adjuvant HIPEC in high-risk CRC patients. The most appropriate patient selection criteria and optimal timing of adjuvant HIPEC have still to be defined.

\section{CONFLICT OF INTEREST}

The authors declare no conflict of interest. 


\section{REFERENCES}

Andre T, Boni C, Navarro M, Tabernero J, Hickish T, Topham C, Bonetti A, Clingan P, Bridgewater J, Rivera F, de Gramont A (2009) Improved overall survival with oxaliplatin, fluorouracil, and leucovorin as adjuvant treatment in stage II or III colon cancer in the MOSAIC trial. J Clin Oncol 27(19): 3109-3116.

Brodsky JT, Cohen AM (1991) Peritoneal seeding following potentially curative resection of colonic carcinoma: implications for adjuvant therapy. Dis Colon Rectum 34(8): 723-727.

Catalano V, Loupakis F, Graziano F, Torresi U, Bisonni R, Mari D, Fornaro L, Baldelli AM, Giordani P, Rossi D, Alessandroni P, Giustini L, Silva RR, Falcone A, D’Emidio S, Fedeli SL (2009) Mucinous histology predicts for poor response rate and overall survival of patients with colorectal cancer and treated with first-line oxaliplatin- and/or irinotecan-based chemotherapy. Br J Cancer 100(6): 881-887.

Chouillard E, Ata T, De JB, Maggiori L, Helmy N, Coscas Y, Outin H (2009) Staged laparoscopic adjuvant intraperitoneal chemohyperthermia after complete resection for locally advanced colorectal or gastric cancer: a preliminary experience. Surg Endosc 23(2): 363-369.

Chu DZ, Lang NP, Thompson C, Osteen PK, Westbrook KC (1989) Peritoneal carcinomatosis in nongynecologic malignancy. A prospective study of prognostic factors. Cancer 63(2): 364-367.

Dutch Cochrane Center. Checklist for cohort studies (2014a) http://dcc. cochrane.org/sites/dcc.cochrane.org/files/uploads/cohort.pdf.

Dutch Cochrane Center. Checklist for randomised controlled trials (RCT) (2014b) http://dcc.cochrane.org/sites/dcc.cochrane.org/files/uploads/ RCT.pdf.

Elias D, Bonnay M, Puizillou JM, Antoun S, Demirdjian S, El OA, Pignon JP, Drouard-Troalen L, Ouellet JF, Ducreux M (2002) Heated intra-operative intraperitoneal oxaliplatin after complete resection of peritoneal carcinomatosis: pharmacokinetics and tissue distribution. Ann Oncol 13(2): 267-272.

Elias D, Honore C, Dumont F, Ducreux M, Boige V, Malka D, Burtin P, Dromain C, Goere D (2011) Results of systematic second-look surgery plus HIPEC in asymptomatic patients presenting a high risk of developing colorectal peritoneal carcinomatosis. Ann Surg 254(2): 289-293.

Elias D, Lefevre JH, Chevalier J, Brouquet A, Marchal F, Classe JM, Ferron G, Guilloit JM, Meeus P, Goere D, Bonastre J (2009) Complete cytoreductive surgery plus intraperitoneal chemohyperthermia with oxaliplatin for peritoneal carcinomatosis of colorectal origin. J Clin Oncol 27(5): 681-685.

Franko J, Shi Q, Goldman CD, Pockaj BA, Nelson GD, Goldberg RM, Pitot HC, Grothey A, Alberts SR, Sargent DJ (2012) Treatment of colorectal peritoneal carcinomatosis with systemic chemotherapy: a pooled analysis of north central cancer treatment group phase III trials N9741 and N9841. J Clin Oncol 30(3): 263-267.

Gesson-Paute A, Ferron G, Thomas F, de Lara EC, Chatelut E, Querleu D (2008) Pharmacokinetics of oxaliplatin during open versus laparoscopically assisted heated intraoperative intraperitoneal chemotherapy (HIPEC): an experimental study. Ann Surg Oncol 15(1): 339-344.

Glehen O, Kwiatkowski F, Sugarbaker PH, Elias D, Levine EA, De SM, Barone R, Yonemura Y, Cavaliere F, Quenet F, Gutman M, Tentes AA, Lorimier G, Bernard JL, Bereder JM, Porcheron J, Gomez-Portilla A, Shen P, Deraco M, Rat P (2004) Cytoreductive surgery combined with perioperative intraperitoneal chemotherapy for the management of peritoneal carcinomatosis from colorectal cancer: a multi-institutional study. J Clin Oncol 22(16): 3284-3292.

Graf W, Westlin JE, Pahlman L, Glimelius B (1994) Adjuvant intraperitoneal 5-fluorouracil and intravenous leucovorin after colorectal cancer surgery: a randomized phase II placebo-controlled study. Int J Colorectal Dis 9(1): 35-39.

Hompes D, Tiek J, Wolthuis A, Fieuws S, Penninckx F, Van CE, D'Hoore A (2012) HIPEC in T4a colon cancer: a defendable treatment to improve oncologic outcome? Ann Oncol 23(12): 3123-3129.

Honore C, Goere D, Souadka A, Dumont F, Elias D (2013) Definition of patients presenting a high risk of developing peritoneal carcinomatosis after curative surgery for colorectal cancer: a systematic review. Ann Surg Oncol 20(1): 183-192.

Jayne DG, Fook S, Loi C, Seow-Choen F (2002) Peritoneal carcinomatosis from colorectal cancer. Br J Surg 89(12): 1545-1550.
Kelsen DP, Saltz L, Cohen AM, Yao TJ, Enker W, Tong W, Tao Y, Bertino JR (1994) A phase I trial of immediate postoperative intraperitoneal floxuridine and leucovorin plus systemic 5-fluorouracil and levamisole after resection of high risk colon cancer. Cancer 74(8): 2224-2233.

Klaver YL, Simkens LH, Lemmens VE, Koopman M, Teerenstra S, Bleichrodt RP, de Hingh IH, Punt CJ (2012) Outcomes of colorectal cancer patients with peritoneal carcinomatosis treated with chemotherapy with and without targeted therapy. Eur J Surg Oncol 38(7): 617-623.

Kopetz S, Chang GJ, Overman MJ, Eng C, Sargent DJ, Larson DW, Grothey A Vauthey JN, Nagorney DM, McWilliams RR (2009) Improved survival in metastatic colorectal cancer is associated with adoption of hepatic resection and improved chemotherapy. J Clin Oncol 27(22): 3677-3683.

Koppe MJ, Boerman OC, Oyen WJ, Bleichrodt RP (2006) Peritoneal carcinomatosis of colorectal origin: incidence and current treatment strategies. Ann Surg 243(2): 212-222.

Lemmens VE, Klaver YL, Verwaal VJ, Rutten HJ, Coebergh JW, de Hingh IH (2011) Predictors and survival of synchronous peritoneal carcinomatosis of colorectal origin: a population-based study. Int J Cancer 128(11): 2717-2725.

Los G, Mutsaers PH, Ruevekamp M, McVie JG (1990) The use of oxaliplatin versus cisplatin in intraperitoneal chemotherapy in cancers restricted to the peritoneal cavity in the rat. Cancer Lett 51(2): 109-117.

Lygidakis NJ, Patil A, Giannoulis K, Fukuda T, Kumar R (2010) Laparoscopic hyperthermic intraperitoneal chemotherapy as adjuvant modality following radical surgery for advanced rectal cancer a new look to an old problem. Hepatogastroenterology 57(97): 73-75.

Nordlinger B, Rougier P, Arnaud JP, Debois M, Wils J, Ollier JC, Grobost O, Lasser P, Wals J, Lacourt J, Seitz JF, Guimares dos SJ, Bleiberg H, Mackiewickz R, Conroy T, Bouche O, Morin T, Baila L, Van CE, Bedenne L (2005) Adjuvant regional chemotherapy and systemic chemotherapy versus systemic chemotherapy alone in patients with stage II-III colorectal cancer: a multicentre randomised controlled phase III trial. Lancet Oncol 6(7): 459-468.

Noura S, Ohue M, Seki Y, Yano M, Ishikawa O, Kameyama M (2009) Long-term prognostic value of conventional peritoneal lavage cytology in patients undergoing curative colorectal cancer resection. Dis Colon Rectum 52(7): 1312-1320.

Noura S, Ohue M, Shingai T, Kano S, Ohigashi H, Yano M, Ishikawa O, Takenaka A, Murata K, Kameyama M (2011) Effects of intraperitoneal chemotherapy with mitomycin $\mathrm{C}$ on the prevention of peritoneal recurrence in colorectal cancer patients with positive peritoneal lavage cytology findings. Ann Surg Oncol 18(2): 396-404.

Palermo JA, Richards F, Lohman KK, Lovelace JV, Atkinson J, Case LD, White DR, Blackstock AW (2000) Phase II trial of adjuvant radiation and intraperitoneal 5-fluorouracil for locally advanced colon cancer: results with 10-year follow-up. Int J Radiat Oncol Biol Phys 47(3): 725-733.

Panteix G, Guillaumont M, Cherpin L, Cuichard J, Gilly FN, Carry PY, Sayag A, Salle B, Brachet A, Bienvenu J (1993) Study of the pharmacokinetics of mitomycin $\mathrm{C}$ in humans during intraperitoneal chemohyperthermia with special mention of the concentration in local tissues. Oncology 50(5): 366-370.

Pelz JO, Chua TC, Esquivel J, Stojadinovic A, Doerfer J, Morris DL, Maeder U, Germer CT, Kerscher AG (2010) Evaluation of best supportive care and systemic chemotherapy as treatment stratified according to the retrospective peritoneal surface disease severity score (PSDSS) for peritoneal carcinomatosis of colorectal origin. BMC Cancer 10: 689.

Rekhraj S, Aziz O, Prabhudesai S, Zacharakis E, Mohr F, Athanasiou T, Darzi A, Ziprin P (2008) Can intra-operative intraperitoneal free cancer cell detection techniques identify patients at higher recurrence risk following curative colorectal cancer resection: a meta-analysis. Ann Surg Oncol 15(1): 60-68.

Sadeghi B, Arvieux C, Glehen O, Beaujard AC, Rivoire M, Baulieux J, Fontaumard E, Brachet A, Caillot JL, Faure JL, Porcheron J, Peix JL, Francois Y, Vignal J, Gilly FN (2000) Peritoneal carcinomatosis from non-gynecologic malignancies: results of the EVOCAPE 1 multicentric prospective study. Cancer 88(2): 358-363.

Sammartino P, Sibio S, Biacchi D, Cardi M, Accarpio F, Mingazzini P, Rosati MS, Cornali T, Di GA (2012) Prevention of peritoneal metastases from colon cancer in high-risk patients: preliminary results of surgery plus prophylactic HIPEC. Gastroenterol Res Pract 2012: 141585.

Scheithauer W, Kornek GV, Marczell A, Karner J, Salem G, Greiner R, Burger D, Stoger F, Ritschel J, Kovats E, Vischer HM, Schneeweiss B, 
Depisch D (1998) Combined intravenous and intraperitoneal chemotherapy with fluorouracil + leucovorin vs fluorouracil + levamisole for adjuvant therapy of resected colon carcinoma. Br J Cancer 77(8): 1349-1354.

Segelman J, Granath F, Holm T, Machado M, Mahteme H, Martling A (2012) Incidence, prevalence and risk factors for peritoneal carcinomatosis from colorectal cancer. Br J Surg 99(5): 699-705.

Seymour MT, Trigonis I, Finan PJ, Halstead F, Dunham R, Wilson G, Farrugia D, Chester JD, King J, Brown CB, Slevin ML, Joel SP (2008) A feasibility, pharmacokinetic and frequency-escalation trial of intraperitoneal chemotherapy in high risk gastrointestinal tract cancer. Eur J Surg Oncol 34(4): 403-409.

Speyer JL, Sugarbaker PH, Collins JM, Dedrick RL, Klecker Jr RW, Myers CE (1981) Portal levels and hepatic clearance of 5-fluorouracil after intraperitoneal administration in humans. Cancer Res 41(5): 1916-1922.

Sugarbaker PH, Gianola FJ, Speyer JL, Wesley R, Barofsky I, Myers CE (1985) Prospective randomized trial of intravenous $\mathrm{v}$ intraperitoneal 5-FU in patients with advanced primary colon or rectal cancer. Semin Oncol 12(3 Suppl 4): 101-111.

Tentes AA, Spiliotis ID, Korakianitis OS, Vaxevanidou A, Kyziridis D (2011) Adjuvant perioperative intraperitoneal chemotherapy in locally advanced colorectal carcinoma: preliminary results. ISRN Surg 2011: 529876.

Vaillant JC, Nordlinger B, Deuffic S, Arnaud JP, Pelissier E, Favre JP, Jaeck D, Fourtanier G, Grandjean JP, Marre P, Letoublon C (2000) Adjuvant intraperitoneal 5-fluorouracil in high-risk colon cancer: a multicenter phase III trial. Ann Surg 231(4): 449-456.
Verwaal VJ, Bruin S, Boot H, van SG, van TH (2008) 8-year follow-up of randomized trial: cytoreduction and hyperthermic intraperitoneal chemotherapy versus systemic chemotherapy in patients with peritoneal carcinomatosis of colorectal cancer. Ann Surg Oncol 15(9): 2426-2432.

Verwaal VJ, van RS, de Bree E, van Sloothen GW, van Tinteren H, Boot H, Zoetmulder FA (2003) Randomized trial of cytoreduction and hyperthermic intraperitoneal chemotherapy versus systemic chemotherapy and palliative surgery in patients with peritoneal carcinomatosis of colorectal cancer. J Clin Oncol 21(20): 3737-3743.

Verwaal VJ, van TH, van RS, Zoetmulder FA (2004) Predicting the survival of patients with peritoneal carcinomatosis of colorectal origin treated by aggressive cytoreduction and hyperthermic intraperitoneal chemotherapy. Br J Surg 91(6): 739-746.

Yang SH, Lin JK, Lai CR, Chen CC, Li AF, Liang WY, Jiang JK (2004) Risk factors for peritoneal dissemination of colorectal cancer. J Surg Oncol 87(4): 167-173.

Yu W, Whang I, Suh I, Averbach A, Chang D, Sugarbaker PH (1998) Prospective randomized trial of early postoperative intraperitoneal chemotherapy as an adjuvant to resectable gastric cancer. Ann Surg 228(3): $347-354$.

This work is published under the standard license to publish agreement. After 12 months the work will become freely available and the license terms will switch to a Creative Commons AttributionNonCommercial-Share Alike 3.0 Unported License.

Supplementary Information accompanies this paper on British Journal of Cancer website (http://www.nature.com/bjc) 